\title{
Modelo predictor de las conductas del balance energético corporal BEC Predict model of the body energy balance BEB behaviors
}

\author{
Andrés Gómez-A costa, Constanza Londoño \\ Universidad Católica de Colombia (Colombia)
}

\begin{abstract}
Resumen. Hace falta evidencia acerca de la sinergia entre los factores psicológicos y contextual es que subyacen a la práctica simultánea de las conductas del balance energético corporal [BEC] (actividad física [AF], alimentación saludable [AS] y sueño de calidad [SC ]). Por ello, se busca identificar si el optimismo disposicional [OD], flexibilidad en regulación emocional [RE], las creencias compensatorias en salud [CCS] y factores ambientales - socioculturales, predicen los dominios conductuales señalados en adultos, a través de un Modelo de Ecuaciones Estructurales [MEE]. Para ello, se dispuso de una investigación explicativa trasversal con una muestra de 300 participantes, que diligenciaron un cuadernillo compuesto por instrumentos psicométricos val idados (uno por cada variable indagada). Los resultados establecieron que la tendenciaa la real ización de las conductas el $B E C$ es predicha por los factores contextuales referidos y las variables psicológicas evaluadas $(R=.54$, val or $p<$ .05). Se valida un modelo hipotético que incorpora el agregado conductual del sueño en la ecuación del BEC, susceptible de modificación e inclusión de nuevas variables.
\end{abstract}

Palabras clave: balance energético corporal; factores psicológicos protectores; factores contextual es protectores; actividad física; alimentación sal udable; calidad del sueño; modelo de ecuaciones estructurales.

\begin{abstract}
There is a lack of evidence about the synergy between the psychological and contextual factors that underlie the simultaneous practice of the behavioral domains of the Body Energy Balance [BEB] (Physical Activity [PA], Healthy Eating [HE] \& Q ual ity Sleep [QS]). Therefore, we seek to identify whether dispositional optimism [D O ], emotion regulation [ER] flexibility, compensatory health beliefs [CHB], and environmental-sociocultural factors, predict behaviors of the body energy balance $[B E B]$ in adults, through a Structural Equation M odel [SEM ]. To do this, cross-sectional explanatory research was available with a sample of 300 participants, who completed a booklet made up of validated psychometric instruments (one for each variable investigated). The results established that the tendency to perform the BEB behaviors is predicted by the contextual factors referred to, and the psychological variables were evaluated $(R=.54, p$-value $<.05)$. A hypothetical model that incorporates the behavioral aggregate of sleep in the BEB equation is validated, subject to modification and inclusion of new variables.
\end{abstract}

Keywords: body energy balance; psychological protective factors; contextual protective factors; physical activity; healthy eating; quality sleep; structured equations model.

\section{Introducción}

El balance energético corporal [BEC], es el estado ideal de equilibrio entre ingesta calórica, recuperación y gasto de energía (Chiquete $\&$ Tolosa, 2013); el BEC es regulado, en principio, por complejos mecanismos neurohormonales útiles para garantizar la transferencia balanceada entre el gasto calórico y la eficiente recuperación (Economos et al., 2015). Sin embargo, también dependetanto de condicionesambientalescomo del comportamiento individual (Atkinson \& Davenne, 2007), que potencian 0 inhiben los procesos de anabolismo y catabolismo (González-Jiménez \& Schmidt, 2012).

Situaciones relacionadas con factores ambientales y

Fecha recepción: 30-10-20. Fecha de aceptación: 01-02-21

Andrés Gómez-Acosta

cagomez434@ ucatolica.edu.co socio-cultural es como la falta de tiempo, las condiciones particulares de los centros urbanos (automatización del trabajo, el transporte mecanizado, el hacinamiento y la falta de espacios adecuados), el trabajo en horarios nocturnoso rotativos, lafaltade opciones paraalimentos balanceados, el permanecer conectado a internet hasta altas horas de la noche, entre otras, son factores que afectan cada vez más en el repertorio conductual de los individuos, y que comprometan prácticas saludables como dormir con calidad, alimentarse adecuadamente y realizar suficienteA ctividad Física [AF] (Anjos \& Silva, 2021) que, en convergenciacon otras conductas de riesgo (como el consumo de sustancias psicoactivas y un afrontamiento inadecuado del estrés), pueden incidir a un desbalance conductual, en el cual la persona ingiere menos energía de la que necesita, o acumula energía que no gasta, en respuesta a la primera ley de la termodinámica (Drapeau et al., 2011), situación que a su vez conlleva a un desbalance en su masa corporal 
(Freire, 2020), a un aumento en la vulnerabilidad a padecer Enfermedades Crónicas No Transmisibles [ECNT] altamente comorbidas, con afectación de la calidad de vida y de la capacidad funcional (Instituto Nacional de Salud [INS], 2015), y a un aumento en las muertes prematuras en poblaciones entre 30 y 70 años en el mundo (World Health Organization [W HO ], 2018).

El análisis de la interrelación entre los factores psicológicosque comprometen las conductas del Balance Energético Corporal [BEC] (asociadas a una pocacalidad del sueño, la alimentación - nutrición desfavorable y el sedentarismo) y susfactores contextuales predisponentes, es imperativo como objeto de investigación, porque con los resultados obtenidos se pueden diseñar y establecer programas de prácticas saludables sostenibles efectivos, así como pensar en estrategias para su mejoramiento (Greenbaum, 2018).

La prevención de dichos comportamientos, a nivel de políticas públicas, ha comprendido la divulgación de información, la sensibilización de las comunidades y la transformación del equipamiento urbano (Condello et al., 2016; Gehl, 2014), entreotrasacciones(O PS, 2015); sin embargo, estas acciones pueden ser más efectivas si afectan positivamente a los predictores psicológicos moduladores de los factores contextuales mencionados, en la medida en que esto incrementa la probabilidad de ejecución sostenida de las conductas saludables deseadas (Sheeran et al., 2017).

Uno de los factores psicológicos que se ha venido vinculando en la literatura reciente es la Regulación Emocional [RE], entendida como laforma de gestión de la expresión, supresión e intensidad de las emociones conforme con los objetivos y el contexto en el cual se desarrolla la situación específica (Gross, 2015); esta permite una mejor gestión de las condiciones físicas y sociales para una AS y AF más consistentes, así como una mejor higiene del sueño (Gómez-A costa\& Londoño-Pérez, 2020). Por otra parte, el 0 ptimismo Disposicional $[0 \mathrm{D}]$, declarado como un rasgo de personalidad que predispone a tener expectativas y comportamientos adaptativos frente a los sucesos (Sheier \& Carver, 2018), predice altos niveles de RE (Jenaabadi et al., 2015) y la realización de conductas saludables (Scheier \& Carver, 2018). Asimismo, las creencias compensatorias en salud [CCS], definidas como pensamientos, convicciones y opiniones, en las que los efectos de un comportamiento no saludable
(Radtke et al., 2014) han sido relacionadas particularmente con alta ingesta calórica y desar rollo de comportamientos saludables como AF pero de forma no sostenida o desbordada (Berli et al., 2014), e incluso comportamientos disfuncionales como inducción del vómito y purga (Amrein et al., 2017; Kronick et al., 2011; Radtke et al., 2014).

Salvo los trabajos deAdamset al., (2016) y Roskoden et al. (2017), no fue posible identificar más estudios que aborden simultáneamente a los tres segmentos conductuales y que reconozcan la compleja interacción con aspectos psicosociales y contextuales determinantes; así mismo, a excepción de los trabajos de Gordon et al. (2016), y de Roskoden et al. (2017), tampoco se evidencia la forma en que factores psicológicos como la flexibilidad en la RE, el OD, las CCS y los factores del entorno físico y sociocultural predicen la ocurrencia de las conductas del BEC. Por otro lado, existen estudios que establecen relaciones entre las creencias disfuncionales (Schneider et al., 2019) o con poca evidencia cientifica (Robbins et al., 2019) acerca del sueño y sus posibles compromisos en la salud, pero no se identifican estudios que evalúen si específicamente las creencias compensatorias afectan lacalidad del sueño, ni investigaciones que evalúen una posible interacción con procesos como la RE y la OD. Por ello, el objetivo del presente estudio es evaluar un modelo hipotético integrador que reafirme la interrelación recíproca y sincrónica de los factores descritos (Figura 1).

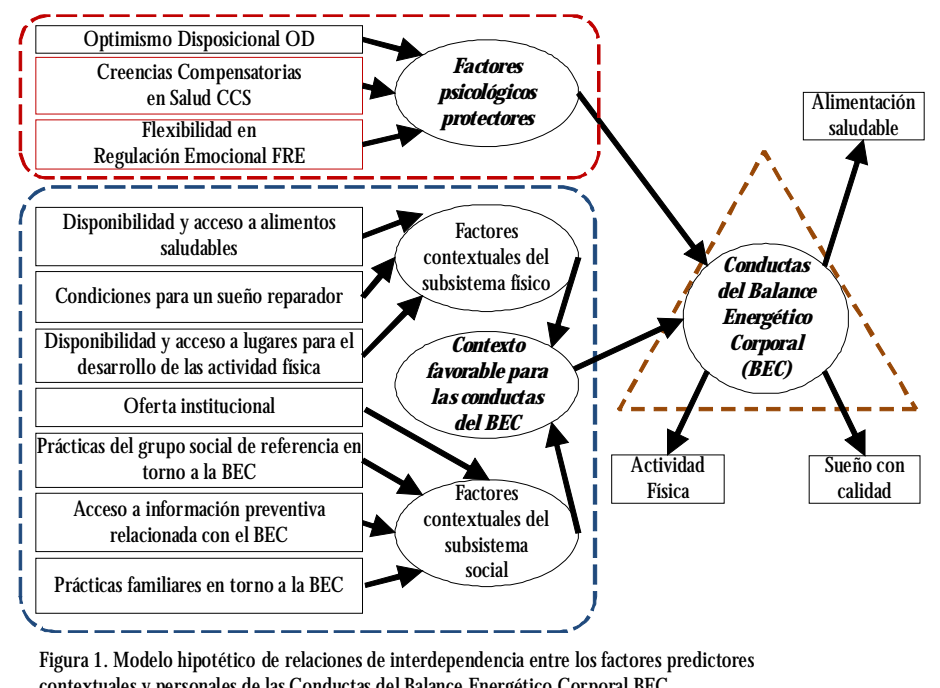

contextuales y personales de las Conductas del Balance Energético Corporal BEC.

\section{Método}

Estudio explicativo con análisis de Modelo de Ecuaciones Estructurales [MEE] para evaluar el modelo hipotético planteado (Hair et al., 2014). 


\section{Participantes}

Fue realizado con 300 adultos, voluntarios (muestreo a conveniencia), de Bogotá - Colombia, sin reporte de enfermedadesfísicas o mentales, cuyo IM C fue calculado a partir de su reporte de peso y talla. De acuerdo con Westland (2010), la muestra cumple con los criterios establecidos para estudios con MEE, (tamaño del efecto $=.25$, potencia estadística $=.80$ y un valor $p<.05$ ). (Tabla 1).

\begin{tabular}{|c|c|c|c|c|c|c|c|}
\hline \multicolumn{7}{|c|}{ Descripción de los participantes de la val idación del MEE predictor de las Conductas el BEC. } & $\%$ \\
\hline Sexo & & & & & Estrato socioeconómico & & \\
\hline Mujer & 186 & 62 & & & 1 & 19 & 6.3 \\
\hline \multirow{2}{*}{ Hombre } & 114 & 38 & & & 2 & 92 & 30.7 \\
\hline & & & & & 3 & 133 & 44.3 \\
\hline Ocupación & & & & & 4 & 42 & 14.0 \\
\hline Sol o estudia & 75 & 25.0 & & & 5 & 10 & 3.3 \\
\hline Solo trabaja & 134 & 44.7 & & & 6 & 4 & 1.3 \\
\hline Estudia y trabaja & 46 & 15.3 & & & & & \\
\hline Desempleado & 25 & 8.3 & & & & & \\
\hline \multirow[t]{2}{*}{ Jubilado } & 20 & 6.7 & & & Nivel académico & & \\
\hline & $M$ & $\mathrm{DE}$ & Mín. & Máx. & Bachillerato & 106 & 35.3 \\
\hline Edad (años) & 34.18 & 14.37 & 18 & 75 & Técnico - tecnólogo & 70 & 23.3 \\
\hline Horas de sueño al día & 6.59 & 1.52 & 1 & 10 & Profesional & 73 & 24.3 \\
\hline IMC (kgs/ mts²) & 24.42 & 3.44 & 17.15 & 37.11 & Posgrado & 31 & 10.3 \\
\hline
\end{tabular}

La muestra es clasificada de acuerdo con el Índice de M asa Corporal [IM C] calculado a partir de los datos de peso y talla reportados; así, se encuentraque 36 personas $(11.80 \%)$ se ubican en bajo paso, $110(36.5 \%)$ en normopeso, 85 (28.4\%) en sobrepeso y 69 (23.3\%) en obesidad. Para el desarrollo del MEE se eliminaron 4 valores extremos, pues no se ajustaban al comportamiento típico de la población; uno de ellos tenía un IM C mayor a 40, otra persona menor a 16, y otros dos participantes eran deportistas de al to rendimiento.

\section{Instrumentos}

Escala de Flexibilidad en Regulación Emocional [FREE Scale, por sus iniciales en inglés] de Bonanno y Burton (2016). Compuesta por 16 ítems y seis opciones de respuesta tipo Likert (que fluctúan desde «Incapaz» - 1 -, a «Muy Capaz» - 6 -), evalúa la expresión y supresión de emociones positivas y negativas ante algunas situaciones sociales típicas (en el contexto de la cultura occidental). De acuerdo con su adaptación para Colombia llevada a cabo conTeoría de Respuesta al Ítem [TRÍ] seidentificaunapruebaunidimensional quereporta una confiabilidad de .98 y un índice de separación de 7.0 (Gómez-A costa, 2020). La sumatoria refiere una puntuación mínima de 16 y un máximo de 76.

Cuestionario de C reencias Compensatorias en Salud [CBHS por sus iniciales en inglés] de Knäuper et al. (2004). Mide creencias asociadas a conductas saludables que intentan compensar conductas no saludables. Su calificación consiste en sumar los puntajes reportados. La versión original consta de 17 ítems $(\alpha=.80)$, mientras que la adaptación desarrollada para Colombia (realizada medianteTRÍ) se compone por 20 ítems, y cinco opciones de respuesta tipo Likert (que van de «Totalmente en Desacuerdo -1, a «Totalmente de Acuerdo» - 5), con una confiabilidad de . 99 y un índice de separación de 10.79 (Gómez-Acosta, 2020). La puntuación total fluctuáentre20 (pocapresenciadeCCS) y 100 (máxima presencia de CCS).

Cuestionario de 0 ptimismo Disposicional [COP] de Velasco, Londoño y Alejo (2014). Permite clasificar la población en tres dimensiones $(O D$, optimistas no realistas y pesimistas). Con 21 ítems, presenta una confiabilidad de $\alpha=.85$. Cada ítem presenta una situación típica con tres opciones de respuesta, las cuales representan una posibilidad pesimista, una optimista disposicional y una optimista no realista; su diligenciamiento implica el registro de una única respuesta por ítem, la cuál se ubica en la categoría que representa, de tal forma que se trabajó únicamente con la sumatoria de los puntajes de OD arrojados por cada participante, con un mínimo de 0 respuestas y un máximo de 21.

Cuestionario de Factores Contextuales de las Conductas del BalanceEnergético Corporal [CFCCBC]. Instrumento Ad H oc diseñado por Gómez-A costa(2020), contiene 30 preguntas referidas a condiciones físicas y sociales promotoras de laAS, laAF y lacal idad del sueño. La calificación del instrumento se ajustó a cada factor medido. Para el caso del contexto físico, se asignó una puntuación de 1 a las opciones de respuesta si, mientras que se designó con 0 a las opciones que referían no presenciao desconocimiento de lacondición preguntada, para una puntuación máxima de 20. Por su lado, para la calificación del contexto social, se puntúa3 alas opciones «la mayoría» 0 «siempre», 2 a «casi la mayoría» 0 «a menudo», 1 a «casi ninguno», «si» 0 «rara vez», y 0 a las opciones «ninguno», «nunca», «no», y «no sée, para una sumatoria máxima de 42 puntos. La dimensión de factores físicos presenta una confiabilidad de . 96 (índice de separación 4.61), mientras que la dimensión de factores sociales .99 (índice de separación 9.00), deacuerdo con GómezAcosta (2020).

Encuesta de Comportamiento Alimentario [ECA] de Ángel et al., (2000). Es una escala unidimensional que describe comportamientos relacionados a la alimentación, la figura y el peso corporal. Presenta una $\alpha=.91$, y su calificación consiste en puntuar de 0 (la opción más adaptativa) a 3 (la opción menos saludable); 
luego de la correspondiente sumatoria, se declara que puntajes cercanos a 0 indicarían un comportamiento al imentario óptimo, mientras que una suma mayor a 24 indica un posible trastorno alimentario.

Cuestionario M undial sobreActividad Física[IPAQ ]. Diseñado por el Departamento de Enfermedades Crónicas y Promoción de la Salud (O MS, 2009), consta de 16 ítemsfrentealoscualeslos respondientes reportan el tiempo estimado (en minutos) deAF (leve, moderada e intensa) que hacen semanalmente en los contextos laboral, hogar, transporte y tiempo libre. Para su puntuación, se multiplica el índice de requerimientos metabólicos MET-minuto correspondiente al tipo de actividad mencionada por el tiempo en minutos registrados en cada una de las categorías (contextos) indagados. LOS MET's de referencia la obtención del coheficiente son 3.3 paracaminar, 4.0 paraAF moderada, y 8. 0 paraAF vigorosa(M antilla\& Gómez-Tolosa, 2007). Luego se hace la sumatoria total de M ET's gastados por semana, y dicho cál culo constituye el indicador numérico de la dimensión AF en este estudio.

Índice de Calidad del Sueño de Pittsburgh, validado por Escobar-Córdoba y Eslava-Schmalbach (2005). Evalúa a partir de 19 ítems factores determinantes de la calidad del sueño que se califican de 0 (óptimo) a 3 (problemático) agrupadosen sietecomponentes: calidad, latencia, duración, eficiencia, perturbaciones, uso de medicamentos y somnolencia diurna; la sumatoria de los componentes refiere un índice que en la medida que se acerca a 0 señala una óptima calidad del sueño, mientras que indicadores cercanos a 21 refieren a atención clínica y tratamiento médico para lograr un sueño restaurador. Este instrumento es unidimensional, y presenta una confiabilidad $\alpha=.78$.

Es preciso anotar que en todas las escalas validadas mediante metodologíaTRÍ con el Modelo de Rasch se observó un comportamiento adecuado en términos de ajuste (validez) de cada uno de los ítems (INFIT y OUTFIT ubicados entre 8 y 1.3), y una correlación ítem - prueba superior a .40, lo cual es considerado óptimo; de igual manera, se aclara que, de acuerdo con Bond y Fox (2007), y Linacre (2002), que índices de separación superiores a 1.5 y una confiabilidad total de la prueba superior a .70 se consideran adecuados.

\section{Procedimiento}

Se proyectó un modelo hipotético y la propuesta metodológica con las consideraciones éticas pertinentes (Ley 1090 de 2006 - Código Deontológico y Bioético del Ejercicio de la Psicología; Resolución № 8430 del 4 de octubre de 1993 del Ministerio de Salud), así como las disposiciones para el tratamiento de datos (Ley 1581 de 2012). El protocolo fue aprobado por el comité de ética de la U niversidad Católica de Colombia (Acta 4 / octubre de 2018).

El acceso a la muestra fue facilitado por el Grupo Empresarial Keralty y CHC Consulting, previas concertaciones con los representantes de dichas entidades, y la aplicación se desarrolló en condiciones óptimas deiluminación, ventilación, privacidad y control de ruidos. Las personas firmaron el consentimiento respectivo, y respondieron alosinstrumentos dispuestos. La captura de datos se hizo mediante el software Excel versión 2016 de la plataforma Microsoft O ffice ${ }^{\circledR}$, con permanente monitoreo del investigador principal.

\section{Análisis de datos}

Se desarrolló un análisis descriptivo, pruebas de normalidad (Kolmogorov-Smirnov), con el software SPSS 24, con una significación estadística menor a 0.05, y una correlación de Pearson paraidentificar la dirección de las posibles relaciones estadísticas entre las variables evaluados. Para establecer el coeficiente de equilibrio de las conductas del BEC se transformaron las puntuaciones obtenidas en puntajes $Z$ (con parámetro de significancia entre 3.0 y -3.0), se invirtieron los puntajes de comportamiento alimentario y calidad del sueño, se realizó la sumatoria de los puntajes normalizados $Z$, y el producto se estandarizó en puntuaciones $T$, de acuerdo con las recomendaciones establecidas para la transformación de puntajes en psicología (Botello, Suero, \& Ximénez, 2012).

El modelo hipotético se evaluó con un M EE real izado con el aplicativo AMOS 25®, según la secuencia planteada por Hair et al. (2014); losindicadores de ajuste que se aplicaron fueron un Chi Cuadrado mayor a .05, una Media de la Raíz Cuadrada del Error por Aproximación (RSMEA) con valores inferiores a .10, un ajuste comparativo de Bender (CFI) superior a .95, y un índice de ajuste normalizado (NFI) mayor a.90.

\section{Resultados}

Los participantes manifestaron bajos niveles de CCS $(M=26.71 ; D E .=9.24)$ con alta dispersión, así como nivelesaltosen flexibilidad en $R E(M=58.14, D E .=8.46)$ y en $O D(M=9.75, D E .=3.36)$. Se identificaron niveles medios de actividad física [AF] con alta dispersión en los registros $(M=4108.25, D E .=6984.64)$, alimentación solo 1.05 por debajo del límite (24.0) que refiere a 
hábitos poco saludables $(M=22.95, D E .=7.92)$, y una calidad de sueño ( $M=5.16, D E .=3.14)$ que indicaleve disfunción (Tabla 2). Posteriormente, se realizó un análisis de Kolmogorov-Smirnov, el cual determinó que las variables tuvieron una distribución normal, con lo cual se procede con los análisis de correlación y el MEE.

Tabla 2.

Estadísticos descriptivos de las variables psicológicas y contextuales incluidas en el Modelo de Factores Predictores de las Conductas del BEC

\begin{tabular}{lcccc}
\hline & Mín. & Máx. & M & DE \\
\hline Edad & 18.00 & 75.00 & 33.89 & 14.08 \\
Índice de Masa Corporal IMC (kgs/ mts $\left.{ }^{2}\right)$ & 17.15 & 37.11 & 24.42 & 3.44 \\
Factores del Ambiente Físico reportados & 8.00 & 20.00 & 15.41 & 2.64 \\
Factores del Ambiente Social reportados & 9.00 & 36.00 & 18.97 & 4.74 \\
Flexibilidad en la Regulación Emocional & 29.00 & 78.00 & 58.14 & 8.46 \\
Optimismo Disposicional & 3.00 & 18.00 & 9.75 & 3.36 \\
Creencias Compensatorias & 5.00 & 58.00 & 26.71 & 9.24 \\
Actividad Física (METS Minutos X Semana) & 0.00 & 47093.00 & 4108.25 & 6984.64 \\
Comportamiento Alimentario & 10.00 & 55.00 & 22.95 & 7.69 \\
Calidad del Sueño & 0.00 & 18.00 & 5.16 & 3.14 \\
\hline
\end{tabular}

Al llevar acabo lacorrelación de Pearson, seencontró que tanto las variables psicológicas como los factores contextuales físicos y sociales precursores de las conductas del BEC, presentan correlacionessignificativas con los comportamientos medidos (Tabla 3).

Tabla 3.

Correlaciones entre las variables incluidas en el M odelo de Factores Predictores de las Conductas del BEC

\begin{tabular}{|c|c|c|c|}
\hline & $\begin{array}{c}\text { Actividad } \\
\text { Física }\end{array}$ & $\begin{array}{c}\text { Alimentación } \\
\text { Saludable }\end{array}$ & $\begin{array}{c}\text { Calidad del } \\
\text { Sueño }\end{array}$ \\
\hline Edad (años) & $-.116^{*}$ & -.084 & .093 \\
\hline Sexo & $-.139 *$ & $-.175^{* *}$ & $-.201 * *$ \\
\hline Índice de M asa Corporal (IMC) & $-.129 *$ & $-.254^{* *}$ & $-.183^{* *}$ \\
\hline Factores del Ambiente Físico reportados & $.453^{* *}$ & $.418^{* *}$ & $.360^{* *}$ \\
\hline Factores del Ambiente Social reportados & $.453^{* *}$ & $.434 * *$ & $.202 * *$ \\
\hline Flexibilidad en Regulación Emocional & $.700^{* *}$ & $.583 * *$ & $.350^{* *}$ \\
\hline O ptimismo Disposicional & $.430 * *$ & $.370^{* *}$ & $.221^{* *}$ \\
\hline Creencias Compensatorias & $-.548^{* *}$ & $-.569 * *$ & $-.370^{* *}$ \\
\hline
\end{tabular}

de AF (.41), AS (.67) y sueño con calidad (.62). La tabla 4 reporta que el modelo presentó un ajuste adecuado, de acuerdo con los criterios definidos para esta metodología de análisis (Hair et al., 2014).

\begin{tabular}{|c|c|c|}
\hline Índices de ajuste & $\begin{array}{l}\text { MEE Equilibrio } \\
\text { Conductas BEC }\end{array}$ & $\begin{array}{l}\text { Parámetros } \\
\text { Esperados }\end{array}$ \\
\hline Razón Chi cuadrado/ grados de libertad & 2.11 & $<3.0$ \\
\hline Raíz Cuadrada del Error Cuadrático M edio (RSMEA) & .06 & $<.10$ \\
\hline Índice de Ajuste Normalizado (NFI) & .99 & $>.90$ \\
\hline Índice Comparativo deAjuste (CFI) & .99 & $>.95$ \\
\hline Índice no Normal izado deAjuste (TLI) & .97 & $>.90$ \\
\hline Chi cuadrado & 10.55 & $\ldots$ \\
\hline Val or $p$ del Chi cuadrado & .06 & $>.05$ \\
\hline Varianza explicada $\left(R^{2}\right)$ & .54 & ... \\
\hline
\end{tabular}

\section{Discusión}

En cuanto a los análisis descriptivos, se puede observar que el IMC calculado ubica a los participantes en el límite crítico entre normopeso y sobrepeso, lo cual indica que estos deben ejecutar de forma sostenida las conductas del BEC para no inducir una acumulación de la energía (Freire, 2020), que a largo plazo influya en el padecimiento de ECNT (Harrington \& LeeChiong, 2013). Por otro lado, los indicadores negativos significativos entre IMC y las conductas del BEC son coherentescon el sedentarismo (Chennaoui et al., 2015), dietasinadecuadas(Fisher, 2014), y bajacalidad del sueño (Unger \& 0 ertel, 2013).

Losparticipantes perciben escenariosapropiadospara el desarrollo deAF (Condello et al., 2016; Gehl, 2014), la obtención, preparación y consumo de alimentos

EI MEE obtenido que mejor se ajustó alas posibilidades teóricas y observadas (figura 2) demostró que la presenciasimultánea de las tres conductas del BEC (.54) es predicho por las variables contexto físico favorable (.28), contexto social favorable (.15), flexibilidad en RE (.28), OD (.06), y CCS $(-.22)$. Se aprecia ademásquelatendencia al equilibrio de las conductas del BEC (variable latente) es explicadapor lavarianza

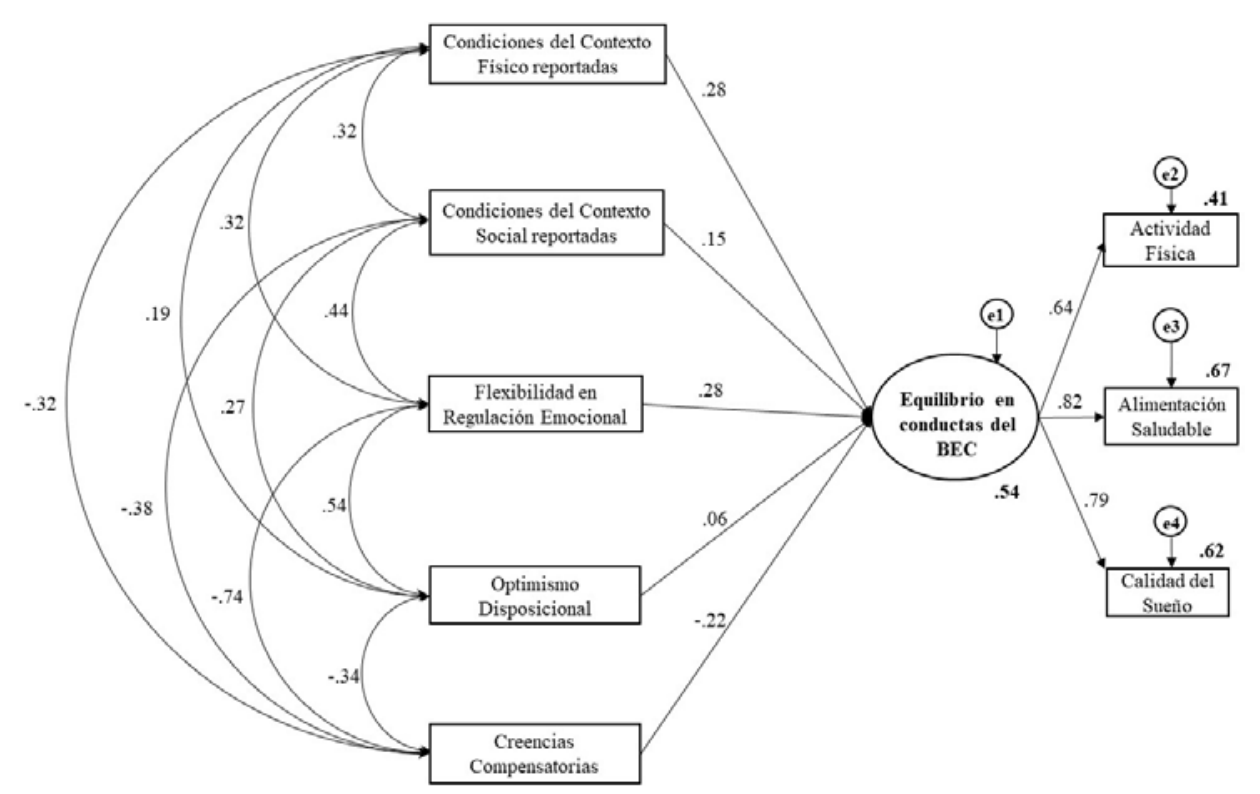

Nota: Todos los pesos de varianza presentan una $p=0.05$.

Figura 2. MEE predictor de las conductas que conforman el BEC 
saludables (Phelan \& Foster, 2014), y control de los estímulos para un sueño con calidad (Harrington \& LeeChiong, 2013). Esto aportó a la predicción de las conductas del modelo, lo cual concuerda con lo evidenciado por Bauer et al. (2011), y M uñoz-Rodríguez et al. (2018); si los individuos identifican posibilidades reales de su contexto físico y social, cobra importancia para ellos la información relativa a la ejecución sostenida de comportamientos saludables (Rogala, 2017), y se promueve el uso de sus propios recursos psicológicos para afrontar posibles barreras como aquellas subyacentes al aprovechamiento de los escenarios disponibles para la práctica deAF (Gardner et al. , 2015; K wasnicka et al., 2016), AS (Freire, 2020) y un sueño de calidad (Irish et al., 2014), incluso en la niñez y adolescencia(Vidarte-Clarosetal., 2021). Por otro lado, al observar las variables psicológicas indagadas, los participantes manifiestan niveles medio - altos de los constructos flexibilidad en RE y OD, así como valores medios - bajos en creencias compensatorias en salud [CCS]; esto significa que los participantes tienden a ser competentes en el ajuste de sus emociones (Burton $\&$ Bonanno, 2016), a realizar comportamientos saludables si cuentan con las competencias (Sheier $\&$ Carver, 2018) y a presentar pocas conductas compensatorias (Amrein et al., 2017; Radtke et al., 2014).

Mientras, las correlaciones negativas significativas entre IMC y las conductas del BEC son coherentes con el sedentarismo (Chennaoui et al., 2015), dietas inadecuadas (Fisher, 2014), y baja calidad del sueño (Unger \& 0 ertel, 2013). Estos eventos podrían ser mitigados en la medida en que las personas cuenten con losrecursospsicológicosy perciban factores contextuales favorables para agenciar exitosamente las conductas esperadas (Bandura, 2001). Por lo demás, las correlaciones evidenciadas permitieron inferir que era plausible la tendencia a identificar que el MEE correspondiera con lo planteado en el modelo hipotético.

Ya con referencia al modelo obtenido, se puede considerar que la presencia de los factores psicológicos (en particular la flexibilidad en RE), aún ante un nivel alto de CCS, pueden contribuir al aprovechamiento eficiente del contexto favorablea las conductas del BEC; en consecuencia, emerge lahipótesisquesi losindividuos reportan al gún cambio súbito en el contexto físico o social, el capital psicológico desarrollado podría activar estrategias sustitutivas para mantener una práctica consistente de los comportamientos referidos, sin incurrir en conductas compensatorias (Frayn etal. , 2018); no obstante, se requerirá mayor evidencia empírica que permita confirmar o refutar a futuro esta afirmación.

Se identifica que el contexto social de los participantes (familiar, pares, medios de comunicación y políticas institucionales) evaluado con los ítems de la dimensión social del CFCCBC tiene a emitir repuestas positivas ante opiniones, modelos, expectativas, estados emocionales y evaluaciones de las conductas saludables $A F, A S$ y SC, y potencialmente refuerza los comportamientos del BEC señalados (Gardner et al., 2015); no obstante, esto es posible solo si existe una sincronía entre las relaciones de los miembros de la sociedad, las disposiciones establecidas por los sectores (público y privado) en los que se desenvuelven las personas (Braveman et al., 2011), la coherencia entre la información promocional dispuesta y lo sugerido por los modelos a seguir en los medios de comunicación y redes sociales (López-Carril et al., 2019), los lineamientos estatales para la garantía de la reducción sistemática de inequidades en los determinantes desde una perspectiva socioecológica ( $\mathrm{H}$ offman et al., 2015; Lounsbury \& Mitchell, 2009; Sallis et al., 2008), y el desarrollo de acciones fortalecedoras de los factores intrínsecos (particularmente los psicológicos) para facilitar tanto la mayor motivación individual, como el empoderamiento de las personas en el apoyo colectivo y la optimización del intercambio entre los factores proximales y distales en función de la realización de las conductas descritas (Folta et al. , 2019; van Kasteren et al., 2020). Sólo así se puede contribuir tanto a la «normalización» de dichas acciones en el contexto social en el cual las personas se desenvuelven (K wasnicka et al., 2016), como al reconocimiento de los beneficios que tiene el desarrollo de dichas prácticas en la salud y bienestar (M artins et al., 2019).

Así, una mayor capacidad de RE (en coexistencia con las demás variables) contribuiría a un mejor autocontrol del comportamiento en ambientes obesogénicos con fácil acceso a al imentos hipercalóricos (Giuliani \& Berkman, 2015; Stok et al., 2017), expresado en la reducción de la hiperfagia ( $M$ artínez-Selva $\&$ Sánchez, 2016), una mejor gestión de alimentos con sabores poco palatables (0 'Connor et al, 2011), del consumo de agua pura (Iglesias-Rosado et al., 2011), e incluso, contribuir en la reconfiguración de los hábitos alimentarios en función de la reevaluación de la información contextual ante emociones negativas (Giuliani \& Berkman, 2015). Asimismo, permite la ejecución sostenida de AF (Gardner et al., 2015; Kwasnicka et al., 2016) y una mejor toma de decisiones adecuadas en el desarrollo de la práctica misma 
(Almonacid-Fierro et al., 2020), en la medida en que las personas estén dispuestas a asumir un protocolo de AF (Wollenberg et al., 2015), y existan las condiciones físicas (representadas fundamentalmente en equipamiento urbano) y sociales óptimas en el contexto en el que estas se desenvuelven (Anjos \& Silva, 2021). También podría aportar en incrementar la tendencia a realizar las acciones que permitan un sueño recuperador, incluyendo tanto el control de los factores ambientales (Irish et al., 2014), como la gestión de posibles situaciones familiares y social es que puedan «competir» con ladisposición agarantizar el horario de sueño (Buxton et al., 2015).

EI OD, en interacción con las demás variables (Gordon et al., 2016; Jennabadi et al., 2015; Tavakoli \& Rahmati, 2015), aporta una varianza baja (.06) a una evaluación objetiva tanto de los estímulos provenientes del contexto físico y social como del impacto de las acciones realizadas frente a dichos inductores (Sheier \& Carver, 2018) y una sensación de control (Sproesser et al., 2015). Por lo tanto, se sugiere revisar con estudios posteriores si este hallazgo es sostenido, y si otros factores psicológicos no incluidos en este estudio coadyuvan más a la varianza total del modelo, lo cual se traduce, en la práctica, en una mejor gestión de los estímulos del contexto físico y social inmediato para el desarrollo sostenido delas conductas sal udables descritas, tanto por parte de la población general (Gómez-A costa, 2018) como de población sometida a altas demandas competitivas (Gómez-M arcos \& Sánchez-Sánchez, 2019).

Las CCS aparecen vinculadas en el modelo con valencia negativa tanto a los comportamientos del BEC como a los demás factores contextuales y psicológicos reportados, lo cual es coherente con investigaciones que muestran cómo las personas pesimistas, o que cuentan con estrategias de RE poco eficientes, suelen reportar imagenes corporales distorsionadas, sedentarismo (Berli et al., 2014) o ejercicio físico extremo no saludable (Frayn et al., 2018) y alta ingesta hipercalórica (Kaklamanou \& Armitage, 2012; Radtke et al., 2014). Esto es coherente en la medida en que la poca presencia de este factor (CCS) puede predisponer a comportamientos «compensatorios» si se percibe un contexto físico o social desfavorable para la realización de las prácticas saludables referidas; por su lado, la presencia de CCS podría aportar en la reducción de la disonancia cognitiva que se presenta al realizar alguno de los agregados conductuales (dormir, hacer AF 0 alimentarse adecuadamente) y realizar otra actividad potencial mente gratificante (Jarbøl et al., 2017), por lo cuál un bajo nivel de CCS favorecería la presencia de los comportamientos señalados de manera más consistente. No obstante, como las creencias en general son fluctuantes y dinámicas (Harvey, 2014), el reporte expuesto acáno necesariamente coincidirácon resultados obtenidosen otros contextos culturales, o no se esperaría que se mantenga idéntico en el largo plazo (Sheeran et al., 2017); la misma observación aplica para la flexibilidad en RE, pues se presume que esta varía en función a situaciones sociales y demandas ambientales distintas (Hu et al., 2014).

En virtud de lo expuesto hasta acá, se espera que el anál isis permitael desarrollo de programasque permitan abordar las CCS para su desmitificación con base en la evidencia (Robbins et al., 2019), así como fortalecer la RE a partir de la formación de recursos para el afrontamiento, del reconocimiento delacorrespondencia entre factores contextuales y grupo de estrategias de RE a implementar, y del incremento de la competencia funcional de procesos psicológicos referidos, que contribuyan ala regulación del cambio comportamental en salud sin estimular la emisión de cada conducta de manera aislada (M ichie et al., 2014), y que incluso formen parte del entrenamiento regular de personas inmersas en entornos altamente demandantes como, por ejemplo, atletas de alto rendimiento (Wagstaff, 2014), fuerzas armadas (Stanley \& Larsen, 2019), 0 personal de rescate (Gärtner et al., 2019), siempre y cuando contemplen un rigor metodológico suficiente que permita el mayor control de variables posible y la evaluación rigurosa del efecto (Eisenberg et al., 2013).

Como limitaciones, se señala que en la forma de obtención de la información (autorreporte) las respuestas se apoyan tanto en los recuerdos como en la estimación subjetiva (Dang et al., 2020), aspecto que no necesariamente puede corresponder con aspectos como el tamaño y cantidad de porciones (Martínez et al. , 2018), los tiempos exactos destinados a conductas complejas como dormir o realizar AF (de M oræes Filho et al., 2021), así con las características contextuales reales. Sumado a esto, el cuadernillo implementado pudo ser extenso paraal gunos participantes, lo que puede aumentar el sesgo en las respuestas.

El modelo se desarrolló con un muestreo intencional, lo cual puede asociarse con coeficientes de correlación y pesos de varianza bajos, así como con la imposibilidad de generalización de los resultados. Es probable que con un muestreo probabilístico más amplio (con parámetros de confiabilidad y error más rigurosos), se encuentren 
correspondencias más ajustadas (Bracamontes del Toro et al., 2018).

El coeficiente de tendencia de las conductas del BEC se calculó con puntuacionesT con unamediade 50 y una desviación estándar de 10, lo cual conlleva a asumir la presencia de las tres conductas simultáneamente, configurando un triángulo equilatero hipotético; no obstante, la ausencia o presencia en niveles al tos de una de las conductas puede sesgar dicha tendencia de forma abrupta. Se sugiere en próximos estudios usar procedimientos estadísticos que permitan tener el perfil de triángulos hipotéticos isósceles (dos conductas presentes y una menos evidente), o escalenos (sin una tendencia de comportamiento en particular), en función a las exigencias individuales de gasto y recuperación energética (Freire, 2020).

Como futuras direcciones de investigación, conviene laincorporación debiomarcadoresque permitan obtener un correlato psicofisiológico preciso (Bonanno \& Burton, 2016). También se sugiere medir aspectos cognitivos relacionados tanto con el razonamiento proposicional como con el razonamiento heurístico, para validar el potencial explicativo de comportamientossaludablesque tendría el modelo evaluado (Houlihan, 2018), registrar la activación cardiovascular, el tiempo trascurrido y las calorías gastadas en AF con el uso de un podómetro 0 acelerómetro (de Moraes Filho et al., 2021; Martorell et al., 2020), realizar registros del comportamiento a imentario en condiciones controladas ( $M$ artínez et al., 2018), calcular el porcentaje de grasa magra a partir de medidas antropométricas (Castro-Porras, et al., 2019), y el registro de la eficiencia del sueño a partir de aplicaciones móviles (Aznar-Díaz et al., 2019; Honary et al., 2019).

\section{Referencias}

Adams, M. ,Kazz, D. \& Shenson, D. (2016).A healthylifestylecomposite measure: Significanceand potential uses PreventiveM ediane, 84, 4147. https:/ / dx. doi.org/ 10.1016/ j.ypmed. 2015.12.005

Almonacid-Fierro,A., M artinez-Romero, M ., \&Almonacid-Fierro, M. (2020). Elementosqueinfluyen en el proceso detomadedecisiones en deportesindividualesdeato rendimiento: un estudio cualitativo (Elementsthat influencetheprocessof decision-making in highperformanceindividual sports: aqualitativestudy). Retos, 38(38), 341-348. https/ / doi.org/ 10.47197/ retosv38i38.73966

Amrein, M., Rackow, P., Inauen, J., Radtke, T., \& Sholz, U. (2017).The roleof Compenstory Health Beliefsin eating behavior change:A mixed method study: Appetite, 116, 1-10. https:/ / dx.doi.org/ 10.1016/ j.appet.2017.04.016

Ángel, L.A.,Vásquez, R., Martínez, L.M., Chavarro, K., \& García, l.
(2000). Revista Col ombiana dePsiquiatría, 29 (1), 29-48. http:// www. scielo.orgco/ pdf/ rcp/ v29n1/ v29n1204.pdf

Anjos,V., \& Silva, J. (2021). Políticadepromoção dasaúdeno lazer em academias públicas de Campo Grande - MS, Brasil (Health promotion policy in leisurein publicgymsin Campo Grande-MS, Brazil) (Políticade promoción delasalud del ocio en gimnasios públicosen Campo Grande. Retos (39), 379-387. https/ / doi.org/ $10.47197 /$ retos.V0i39.79382

Atkinson, G.\&Davenne, G. (2007). Relationshipsbetweensleep, physicd activity and human health. Physiology \& Behavior, 90, 229-235. doi: 10.1016/ j.physoeh.2006.09.015

Aznar-Díaz, I., Cáceres-Reche, M.P.,Trujillo-Torres,J. M., \& RomeroRodríguez,J. M. (2019). Impacto delasappsmóvilesen laactividad físca: un meta-análisis(Impactof mobileappson physical activity:A metaranalysis). Retos, 36 (36), 52-57. https:/ / doi.org/ 10.47197/ retos.v36i36.66628

Bandura,A., (2001). Social cognitivetheory:A n agentic perspective. Annual Review of Psychology,52, 1-26. https: / doi.org/ 10.1111/ 1467-839X.00024

Bauer, G., Brink, M., O mlin, S. (2011). Effectsofnoisefrom non-trafficrelated ambient sourceson seep: review of theliterature of 19902010. Noise Health, 13(53):299-309. https:// www.noiseandheatth.org/ text.asp?2011/ 13/53/ 299/82963

Berli, C., Loretini, P., Radtke,T., Hornung, R., \& Scholz, U. (2014). Predicting physical activity in adolescents. Theroleof compensatory health beliefswithin thehealth action processapproach. Psychology \& H ealth, 29(4), 458e474. http:// dx. doi.org/ 10.1080/ 08870446.2013 .865028

Bonanno, G. \& Burton, C. (2016). Regulatory Flexibility:Anindividual differencesperspectiveon copingand emotion regulation. Perspectives on Psychological Science8(6), 591-612. https/ / dx.doi.org/ 10.1177/ 1745691613504116

Bond, T. G. \& Fox, C. M. (2007). Applying the Rasch mode: Fundamental measurementin thehuman sciences(2aed.). Mahwah, NJ: Lawrence Erlbaum

Botello,J., Suero, M., \& Ximénez, C. (2012). AnálisisdeD atosen Psicología I. Madrid: EdicionesPirámide.

BracamontesdelToro, H., Housni, F., Guiza, I.,Aguiera Cervantes, V., Barragán-Carmona, M., eta., (2018). El razonamiento probabilísico enelestudiodel comportamiento dimentario. EnA., López-Espinoza, A., Martínez, y V. AguileraCervantes (Eds). Invesigaciones en ComportamientoAlimentario: Reflexionesalcancesy retos (pp. 201-234). México: PurrúaPrint. http:/ / www.cusur.udg mx/ es/ sites/ default/ files investigaciones_en_comportamento_adimentario.pdf

Braveman, P., Egerter, S., \&W illams, D. (2011).TheSocial Determinants of Health: Coming ofAge. Annual Review of PublicH ealth, 32, 381398. doi: 10.1146/ annurev-publheatth-031210-101218.

Buxton, O., ChangA.M., Sppilsoury,J., Boss,T., Emsellem, H., \&Knutson, K. (2015). Sleepinthemodernfamily: Protectivefamily routines for child and adolescent sleep. Sleep H ealth. https:/ / dx. doi.org/ 10.1016/ j.seh.2014.12.002

Castro-Porras, L., Rojas-Russell, M.,Villanueva-Sánchez, J. , \& LópezCervantes, M. (2019).An anthropometry-based equation offat mass percentage asavalid discriminator of obesity. Public $\mathrm{H}$ ealth Nutrition 22(7):1250-1258. https:/ / dx. doi.org/ 10.1017/ 
S1368980018004044.

Chennaoui, M., Arna, P., Sauvet, F., \& Leger, D. (2015). Sleep and exercise:A reciprocal issue?Sleep M edianeReview, 20, 59-72. https/ / dx. doi.org/ 10.1016/ j.smrv.2014.06.008.

Chiquete, E. \&Tolosa, P. (2013). Conceptostradicionalesyemergentes sobreel balanceenergético. Revisa deEndocrinología y N utrición, 21 (2), 59-68.

Condello, G., Ling, F., Bianco,A., Chasting, S., Cardon, G., etal., (2016). Using concept mapping in the development of the EU-PAD framework (European-Physical Activity Determinantsacrossthe lifecourse): aDEDIPAC-study. BM C PublicH ealth, 16, 1145. https/ / dx.doi.org/ 10.1186/ s12889-016-3800-8.

Dang, J., King, K., \& Inzlicht, M. (2020). Why are self-report and behavioral measuresweakly correlated?Trendsin CognitiveSciences, 24(4), 267-269. https/ / dx.doi.org/ 10.1016/ j.tics 2020.01.007.

deM oræsFilho,J.,Villarrasa-Sapiña, I., Marco-Ahulló,A.,GarcíaM assó, X., \& González, L. -M. (2021). Nuevasperspectivasmetodológicas en validación deacelerómetrosparaestimar laA ctividad Físicade adultosen actividadescuotidianas(N ew methodological approach in accelerometer validation to estimatePhysical Activity of adultsin daily activities). Retos (40), 216-223. https/ / doi.org/ 10.47197/ retos. v1i40.74360

Departamento deEnfermedades Crónicasy Promoción delaSalud (2009). Cuestionario M undial sobreActividad Físca. Recuperado de http:/ / www. who. int/ chp/ steps/ instrument/ es/

Drapeau,V., Hetherington, M., \&Tremblay,A. (2011). Impactof eating and lifestylebehaviorson body weight: beyond energyvalue. EnV., Preddy, R.,Wason \& C. M artin (Eds) H andbook of Behavior,Food and Nutrition,1, (pp.693-706). NewYork: Springer. doi 10.1007/ 978-0-387-92271-3.

Economos, C., Haffield, D., King,A.,Ayda, G., \&Ann-Pentz, M. (2015). Foodand physical activity environment: an energy balanceapproach for research and practice. American Journal of PreventiveM ediane, 48 (5), 620-629. doi: 10.1016/ j.amepre.2014.12.007.

Eisenberg, C., Sánchez-Romero, L., Rivera Dommarco, J., Holub, C., Arredondo, E., Elder, J., \& Barquera, S. (2013). Interventionsto increasephysical activity and healthy eating overweight and obese children in M exico. Salud Pública deM éxico, 55 (Suplemento3) 441446.

Escobar-Córdoba, F. \& Eslava Schmalbach, J. (2005). Validación colombianadel ÍndicedeCalidad deSueño dePittsourgh. Revisa de N eurología 40 (3), 150-155. https:/ / www. researchgate net/ publication/269987211_Colombian_Validation_of the Pittsourg h_Sleep_Q uality_Index_Validacion_colombiana del indice de calidad de sueno de Pittsourgh

Fisher, J. (2014). Healthy diet throughadülthood. EnC. Berdanier, J. Dwyer \& D. Heber (Eds) H andbook of N utrition and Food - Third Edition (pp 337-346). NewYork:Taylor \& FrancisGroup. https:/ / doi.org/ 10.1201/ b15294

Folta, S., Paul, L., Nelson, M., Strogatz, D., Graham, M., Eldridge, G., et al., (2019). Changesin diet and physica activity resultingfrom theStrong $\mathrm{H}$ earts, $\mathrm{H}$ ealthy Communitiesrandomized cardiovascular diseaserisk reduction multilevel intervention trial. International Journal of Behavioral N utrition and Physical Adivity16 (91).doi: 10.1186/ s12966-019-0852-z
Frayn, M., Livshits, S., \& Knäuper, B. (2018). Emotional eding and weight regulation: aqualitativestudy of compensatory behaviors and concerns. Journal of Eating D isorders6 (23), 1-190. https:/ / dx.doi.org/ 10.1186/ s40337-018-0210-6

Freire, R. (2020). Scientific evidenceof dietsfor weightloss Different macronutrientcomposition, intermittentfasting, and popular diets. Nutrition 69, 110549. https:/ / dx. doi. org/ 10.1016/ j.nut. 2019.07.001.

Gardner, B., Smith, L., Lorencatto, F., Hamer, M. \& \& Biddle, S. (2015). How to reducesittingtime?A review of behavior changesstrategies used in sedentary behavior reduction interventionsamongadults Health Psychology Reriew 10(1), 89-112. https/ / dx. doi.org 10.1080/ 17437199.2015.1082146

Gärtner,A., Behnke,A., Conrad, D., Kolassa, I.T., \& Rojas, R. (2019). Emotion Regulation in RescueWorkers: Differential relationship with perceived work-related stressand stress-related symptoms. Frontiers in Psychology, 9, 2744. https:/ / doi.org/ 10.3389/ fpsyg2018.02744

Gehl,J. (2014). Ciudadespara la gente BuenosAires: EdicionesInfinito Giuliani, N. \& Berkman, E. (2015). Craving isan affectivestateand its regulation can beunderstood intermsof theextend processmodel of emotion regulation. Psychological Inquiry, 26 (1), 48-53. https: / dx.doi.org/ 10.1080/ 1047840X.2015.955072.

Gómez-Acosta, C.A. (2018). Factorespsicológicospredictoresdeestilos devidasaludable. RevisadeSalud Pública, 20(2), 155-162. https:/ / doi.org/ 10.15446/ rsap.v20n2.50676

Gómez-Acosta, A. (2020). Identificación de un modelo de predictores de conductasasociadasal balanceenergético corporal en población bogotana mediante modelamiento de ecuaciones estrucurales (Tesis Doctoral Inédita). Bogotá U niversidad CatólicadeColombia

Gómez-AcostaC.A., \& Londoño-PérezC. (2020). Regulaciónemocional y conductasdebalanceenergético corporal enadultos. Unarevisón deevidencia. Ada Colombiana dePsicología,23(2), 349-382. https/ / doi.org/ 10.14718/ ACP.2020.23.2.14

Gómez-M arcos, G., \& Sánchez-Sánchez, M. (2019). Descripcióny diferencias en las variables psicológicas relacionadas con el rendimiento deportivo detriatletasy paratriatletas(Description and differences in the psychological variables related to sports performanceof triathletesand paratriathletes. Retos, 36(36), 2225. https/ / doi.org/ 10.47197/ retos. V36i36.66973

González-Jiménez, E. \& Schmidt,J. (2012). Regulación delaingesta alimentaria y del balance energético; factores y mecanismos implicados. N utrición Hospitalaria, 27 (6), 1850-1859. doi: 10.3305/ nh.2012.27.6.6099.

Gordon, N., Chesney, S. \& Reiter, K. (2016). Thinking positively: Optimism and emotion regulation predict interpretation of ambiguousinformation. Cogent Psychology, 3: 1195068. https:/ / dx.doi.org/ 10.1080/ 23311908.2016.1195068.

Greenbaum, Z. (2018).A greater rolein nutritional health. M onitor in Psychology:2019TrendsReport.American Psychological Association. NewYork: BPAWorldW ide https:/ / www. apa.org/ monitor/ 2018/ 11/ cover-nutritional-health

Gross,.).(2015). Emotion regulation: currentstatusandfutureprospects Psychological Inquiry. 26 (1), 1-26, doi: 10.1080/ 1047840X.2014.940781. 
Hair,..,Anderson, R., Tatham, R., Black,W. (2014). M ultivariateData Analysis NewYork: PrenticeHall. https:/ / doi.org/ 10.1007/ 9783-642-04898-2 395

Harrington, J. \& Lee-Chiong, T. (2013). Circadian rhythm sleep disorders EnV. Preddy,V.Patel yL. Le(Eds). Handbook of nutrition, diet and sleep (pp. 62-70). Wageningen:Wageningen Academic Publishers. https/ / dx.doi.org/ 10.3920/ 978-90-8686-763-9.

Harvey, . (2014). Health belief and health outcomes En L. Martin\& M. DiM atteo (Eds) The Oxford H andbook of $H$ ealth Communication, Behavior ChangeandTreatmentAdherence. (pp. 177-192). N ewYork: OxfordU niversityPress https/ / dx.doi.org/ 10.1093/ oxfordhb/ 9780199795833.001.0001

Hoffmann, R., Eikemo, T., Kulhanová, I., Kulik, M., Looman, C., Menvielle, G., eta., (2015). 0 besityand thepotential reduction of social inequalities in mortality: evidence from 21 European populations. European Journal of PublicH ealth, 25(5), 849-856, doi: 10.1093/ eurpub/ dkv090.

Honary, M., Bell, B.T., Clinch, S.,W ild, S. E., \& M cN aney, R. (2019). Understandingthe Roleof Healthy Etingand FitnessM MobileApps intheFormation of M aladaptiveEating and ExerciseBehaviorsin Young People.JMIR mH ealth and uH ealth, 7 (6), e14239. https:/ / doi.org/ 10.2196/ 14239

Houlihan, S. (2018). Dua-processmodel sofheatth-related behaviour and cognition: areview of theory. PublicH ealth, 156, 52-59. doi: 10.1016/ j. puhe.2017.11.002.

Hu,T., Zhang, D.,Wang, J., Mistry, R., Ran, G., \&Wang, X. (2014). Relation between emotion regulation and mental health:A meta analysisreview. Psychological Reports:M Measures\& Statistics, 114 (2), 341-362. https/ / dx. doi.org/ 10.2466/ 03.20.PR0.114k22w4

Iglesias-Rosado, C., Villamarino-M arín,A., Martínez,J., Cabrerizo, L., Gargallo, M., eta., (2011). Importanciadel aguaen lahidrazción delapoblación española. Documento FESNAD 2010. Nutrición Hospitalaria 26(1), 27-36. https:/ / dx. doi.org/ 10.3305/ nh.2011.26.1.5167.

Instituto Nacional deSalud (2015). Carga deenfermedad por enfermedades crónicasno transmisiblesy discapacidad en Colombia (InformeT écnico). Bogotá: Imprenta Nacional de Colombia. https:/ / www. minsalud. gov.co/ sites/ rid/ Lists/ BibliotecaDigital/ RIDE/ IA/ INS/ informe-ons5. pdf

Irish, L., Kline, C., Gunn, H., Buysse, D., \& Hall, M. (2014).Theroleof sleep hygienein promoting public health: areview of empirical evidence. Sleep M edicine Reviews, 1, 1-14. https:/ / dx. doi.org/ 10.1016/ j.smrv.2014.10.001.

Jarbøl, D., Larsen, P., Gyrd-Hansen, D., Søndergaard, J., Brant, C., Leppin,A., eta., (2017). Determinantsof preferencesfor lifestyle changesversusmedication and beliefsin ability to maintain lifestyle changes A population-based survey. PreventiveM edicineR eports 6, 66-73. https// dx.doi.org/ 10.1016/ j.pmedr.2017.02.010.

Jenaabadi, H., Ali-A hani, M., \& Sabaghi, F. (2015). Examining the relationship of optimism and emotion regulation strategies with general health among students of U niversity of Sistan and Baluchestan. Health, 7, 865-872. https:/ / dx. doi.org/ 10.4236/ health.2015.77102

Kaklamanou, D.\&Armitage, C. (2012). Testing compensatory health beliefs. Psychology\& H ealth, 27(9), 1062-1074. https/ / dx. doi.org/
10.1080/08870446.2012.662974.

Knäuper, B., Rabiau, M.,Cohen, O.,\&Pariciu,N. (2004). Compenstory health beliefs: Theory and measurement. Psychology and $\mathrm{H}$ ealth, 19(5), 607-624. https:// dx.doi. org/ 10.1080/ 0887044042000196737

Kronick, I.,Auerbach, R., Stich, C., \& Knäuper, B. (2011). Compenstory beliefsand intentionscontributetotheprediction of caloricintzein dieters. Appetite,57 (2), 435-438. https:/ / dx. doi.org/ 10.1016/ j.appet. 2011.05.306

Kwasnicka, D., Dombrowski, S.,W hite, M., \& Sniehotta, F. (2016). Theoretical explanationsfor maintenance of behavior change: a systematic review of behavior theories. H ealth Psychology Review 10(3), 277-296. https:// dx.doi.org/ 10.1080/ 17437199.2016.1151372

Lane,A., Davenport,T., Friesen,A., Beedie, C., Fullerton, C., \& Stanley, D. (2015). How should I regulatemy emotionsif I want to run faster? European Journal of Sport Science. https:/ / dx. doi.org/ 10.1080/ 17461391.2015.1080305

Linacre, J. M. (2002). W hat do Infit and 0 utfit, M ean-square and Standardized mean? Rasch Measurement Transaction, 168, 878. Recuperado de: http:/ / www. Rasch.org/ rmt/ rmt162f.htm).

López-Carril, S., Villamón-H errera, M., \& Añó-Sanz, V. (2019). Conceptualización delosmediossociales: oportunidadesparala gestión del deporte(Conceptualistion of Social M edia: opportunities for SportM anagement). Retos, 36(36), 578-583. https/ / doi.org/ 10.47197/ retos. v36i36.68572

Lounsbury, D. \& M itchel, S. (2009). Introduction to special issueon social ecological approachesto comm unity healthresearch and action. American Journal ofCommunity Psychology,44, 213-220, doi: 10.1007/ s10464-009-9266-.

Mantilla, S. \& Gómez-Tolosa,A. (2007). El Cuestionario Internacional deActividadFísca. Un instrumento adecuado en el seguimiento de laactividad físicapoblacional. Revisa Iberoamericana deFisioterapiay Kinesiología, 10 (1), 48-52. https:/ / dx. doi.org/ 10.1016/ S11386045(07)73665-.

Martínez,A., López-Espinoza, A. \&A guileraCervantes, V. (2018). O bservacióny registro conductual deepisodiosdealimentaciónen humanos Principiosyprocedimientos EnA., López-Espinoza,A., Martínez, y V. Aguilera-Cervantes (Eds). Investigaciones en ComportamientoAlimentario: Reflexionesalcancesy retos (pp. 165-200). México: PurrúaPrint. http:/ / www.cusur.udg mx/ es/ sites/ default/ files investigaciones_en_comportamento_dimentario.pdf Martínez-Selva, J., \& Sánchez, J. (2016). Estados emocionales y preferenciasalimentarias aspectospsicológicosy aplicados. EnA. López-Espinosa\&A. Martínez(Eds). La Educación en Alimentacióny Nutrición (pp. 187-207). México: M cGraw Hill Eduction.

Martins, J., Cabral, M., Eliz, C., Nela, R., Sarmento, H., Marques,A., \& Nicola, P. (2019). Physical activity recommendationsfor health: knowledge and perceptions among college students (Recomendacionesdeactividadfísicaparalasalud: conocimientoy percepcionesentreestudiantesuniversitarios). Retos, 36 (36), 290296. https/ / doi.org/ 10.47197/ retos. v36i36.68324

Martorell, M., Labraña, A., Ramírez-Alacón, K., Díaz-Martínez, X., Garrido-Méndez,A., etal., (2020). Comparación delosnivelesde actividadfísicamedidoscon cuestionario deautorreporte(IPAQ) 
con medición deacelerometríasegún estado nutricional. Revisa MédicadeChile, 2(148), 37-45. https/ / dx. doi.org/ 10.4067/ S003498872020000100037

Michie, S., West, R., Campbell, R., Brown, J., \& Gainforth, H. (2014). $A B C$ of Behaviour ChangeTheories. Sutton, Surrey, UK: Silverback Publishing

Muñoz-Rodríguez, D.,Arango-Alzate, C., \& SeguraCardona,A. (2018). Entornosy actividad físicaen enfermedadescrónicas: másalláde factoresasociados. Universidad y Salud 20(2), 183-199. https:/ I dx. doi.org/ 10.22267/ rus182002.122

O'Connor, D., Jones, F., \& Conner, M. (2011). Psychological stress, diarymethodsand eating behavior. EnV., Preddy, R.,Wason\& C. Martin(Eds). H andbook of Behavior, Food and N utrition, (pp. 16191633). NewYork: Springer. https:/ / dx. doi.org/ 10.1007/ 978-0387-92271-3.

Phelan,S., \& Foster, G. (2014). Environmental ChallengesandAssesment. EnC. Berdanier, J. D wyer \& D. Heber (Eds.) H andbook of N utrition and Food-Third Edition (pp 703-713). N ewYork:Taylor \& Francis Group. https:/ / doi.org/ 10.1201/ b15294

Radtke, ., Kaklamanou, D., Scholz, U., Hornung, R., \&Armitage, C. (2014).A rediet-specific compensatory health beliefspredictive of dieting intentions and behavior? Appetite, 76, 36-43. https:/ / dx.doi.org/ 10.1016/ j.zppet.2014.01.014.

Robbins, R., Grandner, M.A., Buxton, O. M., Hale, L., Buysse, D.J., Knutson, K. L.,Patel, S. R., Troxel,W. M.,Youngtedt,S. D., Czeisler, C.A., \& Jean-Louis, G. (2019). Sleep myths: an expert-led studyto identify falsebeliefsaboutseep thatimpingeupon population seep health practices. Sleep H ealth, 5(4), 409-417. https: / / doi.org/ 10.1016/j.seh.2019.02.002

Rogala, M. (2017). Health promotion actionsin mass mediafor seniors inselected European countries. Epidemiol ogy Biostatisticsand Public H ealth, 14(1), el2410-1. https:/ / dx. doi. org/ 10.2427/ 12410

Roskoden, F., Krüger, J.,Vogt, L., Gärtner, S., Hannich, H., Steveling, A., etal., (2017). Physical activity, energy expenditure, nutritional habits, quality of sleep and stresslevelsin shift-working healthcare personnel. PLOSON E 12(1), 1-21. https:/ / dx.doi.org/ 10.1371/ journal.pone 0169983.

Sall lis,J., O wen, N., \& Fisher, E., (2008). Ecological model sof health behavior. EnK., Glanz, B., Rimer \& K.,Viswanath (Eds). Health behavior and heal th education:Theory, research and practice(4th Edition) (pp. 465-486). SanFrancisco:JosseyBass

Schneider, M., Kovas,Y., \& Gregory,A., (2019). Dysfunctional beliefs aboutseep and insomniasymptomsinearly adulthood:A twin and sibling study. Journal of Sleep Research, 28(1), 28:e12834. https./ doi.org/ 10.1111/ jsr.12834.

Sheeran, P., Klein,W. \& Rothman,A. (2017). Health behavior change: Moving from observation to intervention. Annual Review of Psjchology68(1), 573-600. https/ / dx. doi.org/ 10.1146/ annurevpsych-010416-044007

Sheier, M., \& Carver, C. (2018). Dispositional optimism and physical health:A longlook back, aquick look forward. American Psychologist, 73(9), 1082-1094. https/ / dx.doi.org 10.1037/ amp0000384.

Sproesser, G., Klusmann, V., Schupp, H., \& Renner, B. (2015). Comparativeoptimism abouthealthyeating Appetite, 90, 212-218. https/ / dx. doi.org/ 10.1016/ j.appet.2015.03.008
Stanley,E.A., \&Larsen, K. L. (2019). Difficulties/ $N$ ithEmotionRegultion in the Contemporary U.S.Armed Forces Structura Contributors and Potential Solutions. Armed Forces\& Sociely, 0095327X1984801. doi:10.1177/ 0095327×19848018

Stok, M., Hoffman, S., Volkert, D., Boeing, H., Ensenauer, R. etal., (2017).TheD O NE framework:Creation, evaluation and updating of an interdisciplinary dynamic framework2.0 of determinants of nutrition and eating PLOSONE 12(2): e0171077. doi:10.1371/ journal. pone.0171077.

Tarkoli,F,\& Rahmati,A. (2015). Mediatingroleofoptimismin difficulties in emotion regulation scaleand psychological well-beingamong female students living in the dormitory of U niversity of Isfahan (UI). J ournal of Scientific Research and D evelopment, 2(2), 108-111. http:/ / www.ænsiweb.net/ AEN SIW EB/ rjfh/ rjfh/ 2015/ June/ 476-480.pdf

Unger, M. \&\&O ertel,W. (2013). Gherlin:A gastric peptidelinkingseep and energy balance. EnV. Preddy,V. Patel yL. Le(Eds). Handbook of nutrition, diet and seep (pp. 175-183). Wageningen:Wageningen AcademicPublishers. https/ / dx. doi. org/ 10.3920/ 978-90-8686763-9.

van Kasteren,Y., Lewis, L., \& M æeder,A. (2020). O ffice based physical activity: mapping asocial ecological model approach againstCO MB. BM C PublicH ealth, 20, 163. doi: 10.1186/ s12889-020-8280-1

Velasco, M., Londoño, C., \&Alejo, I. (2014).Validación del cuestionario deoptimismo disposicional usando lateoríaderespuestaal ítem. Diversitas: Perspectivas en Psicología, 10(2), 275-292. http:/ / www.scielo.orgco/ pdf/ dpp/ v10n2/ v10n2207.pdf

VidarteClaros,J,_Arango-Arenæs,A., ParraSánchez, J., \&Velez-Álvverez, C. (2021). Predictores de condición física saludable desde Determinantes Sociales en escolares colombianos: Estudio multicéntrico (Predictorshealthy physica condition from Social Determinantsin Colombian schoolchildren: Multicenter study). Retos(39), 182-186. https/ / doi.org/ 10.47197/ retos Wi39.77610 Westland,J.C. (2010). Lower boundson samplesizein structural equation modeling. Electronic Commerce Research and Applications, 9(6), 476487, https:/ / dx. doi.org 10.1016/ j.elerap.2010.07.003.

W hybrow, S., King, N., \& Stubbs, J. (2011). Exercise, appetite, and energy balance: theinteractionsbetween energy expenditureand intake, and theimplicationsfor weightmanagement. EnV., Preddy, R., Wason \& C. Martin (Eds). H andbook of Behavior, Food and Nutrition,(pp. 1569-1584). NewYork: Springer. https/ / dx.doi.org/ 10.1007/ 978-0-387-92271-3.

Wollenberg, G., Shriver, L., \& Gates, G. (2015). Comparison ofdisorders eatingsymptomsand emotion reguldion difficultiesbetweenfemale collegeathletesand non-athletes Eating Behaviors, 18, 1- 6. https:/ / dx. doi.org/ 10.1016/ j.eatbeh.2015.03.008.

World Health O rganization [W HO ](2018). N oncommunicablediseases country profiles 2018. Geneva: World Health O rganization. Recuperado de: https:/ / www.who.int/ nmh/ publications/ ncdprofiles2018/ en/ 